EPJ Web of Conferences 116, 11002 (2016)

DOI: $10.1051 /$ epjconf/201611611002

(C) Owned by the authors, published by EDP Sciences, 2016

\title{
A discussion of IceCube neutrino events, circa 2015
}

\author{
Arman Esmaili ${ }^{1}$, Andrea Palladino ${ }^{2}$, and Francesco Vissani ${ }^{1,2}$ \\ ${ }^{1}$ Laboratori Nazionali del Gran Sasso, INFN, Assergi, L'Aquila \\ ${ }^{2}$ GSSI, Gran Sasso Science Institute (INFN), L'Aquila
}

\begin{abstract}
IceCube has changed the rules of the game and continues to progress. Their observations are compatible with cosmic neutrinos undergoing 3 flavor oscillations. The topologies of the events have been used to probe ordinary and exotic physics. Still, we need independent confirmations of this assumption; the astrophysical connections heavily rely on speculations (excepting special cases, such as GRB); the amount of prompt events is not known precisely; double bang and/or Glashow resonance events are still to be seen; the energy and the angular distributions are not well-known, even if the simplest picture (isotropic flux, power law distributed in energy) is still compatible with the data. In this talk, we select specific topics concerning expectations, inferences and prospects.
\end{abstract}

\section{Remarks on prompt neutrinos}

Cosmic rays of sufficiently high energies produce charm mesons interacting in the atmosphere. Their decays, differently from those of pion and kaon mesons, are very fast and produce the so called prompt neutrinos. These decays do not suffer any effect of the atmosphere, thus the signal is not yearly modulated. Prompt neutrinos have a peculiar flavor structure, $v_{e} \approx v_{\mu}$ and $v_{\tau} \approx 0$, within $10 \%$. Thus, the signal from charm is rich in $v_{e}$ that are just $\sim 1 / 20$ of the conventional component. The normalization of prompt neutrinos is not precisely predicted, but at few $10 \mathrm{of} \mathrm{TeV}$, they could dominate atmosperic neutrino spectrum making it harder, till $\alpha \sim 2.7$. The analyses of IceCube experiment deplete prompt events coming from above exploiting neutrino-muon correlation, as suggested in [1]. These analyses probed the normalization predicted by [2], but they did not see trace of prompt events. It is interesting that the cosmic neutrino signal has a slope similar (slightly below) $\alpha \sim 2.7$ and it is certainly important that, as claimed by IceCube, that prompt events, even if present, do not explain the bulk of observations. However, this leaves us the task of finding prompt events, or to understand "why IceCube lacks of charm". The answer could be quite simple but it is important to know it.

\section{Expectations on oscillations of cosmic neutrinos}

Assume cosmic neutrinos have been seen. Ordinary 3 neutrinos oscillations modify their fluxes and thanks to IceCube's capability to classify different topologies of events, these type of effects can be probed. We clarify the expectations and compare with the data, discussing residual uncertainties.

This is an Open Access article distributed under the terms of the Creative Commons Attribution License 4.0, which permits unrestricted use, distribution, and reproduction in any medium, provided the original work is properly cited. 

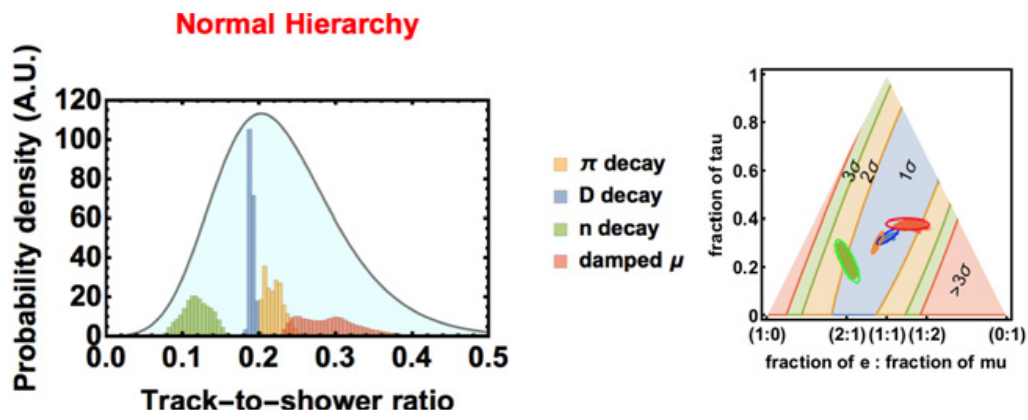

Figure 1. Right panel, the $N_{T} / N_{S}$ (track-to-shower) ratio; the large smooth curve derives from IceCube data analysis, the 4 small histograms are the expectations [4]. Left panel, the flavor triangle; the wide oblique areas are the outcome of data analysis, whereas the 4 dotted areas enclosed by ellipses are the expectations [5]. In both plots it is assumed that the slope of the power spectrum is $\alpha=2.3$ and we consider 4 scenarios for neutrino production mechanism: 1) The (standard) pion decay; 2) the decay of heavy mesons (charm); 3) the neutron decay; 4) the case when the pion decays, but muons are instead trapped in the medium, the damped muon case.

The expectations can be summarized in a few points: 1 . Cosmic neutrinos are supposedly produced in environments with small particle densities. 2. During their propagation, they are subject to vacuum oscillations, also known as oscillations in Gribov-Pontecorvo (averaged) regime. 3. Three flavor oscillations have an important impact on cosmic neutrinos [3] and have been precisely measured in terrestrial laboratories. 4. Residual uncertainties can be treated with MonteCarlo techniques [4] and are most neatly described by adopting the three natural parameters of vacuum oscillations [5].

To date, we have probed the flavor of the incoming neutrinos by measuring two types of event "topologies", namely track events, largely corresponding to charged current muon neutrinos (or antineutrinos) interactions on nucleons and shower events, corresponding to charged $v_{e}, v_{\tau}$ instead, or to neutral currents. The assumption of a power law distribution of the cosmic neutrino fluxes, $F \propto E_{v}^{-\alpha}$ is plausible for a non-thermal population and allows us, along with the effective areas of IceCube, to compute the expected number of tracks and showers, $N_{T}$ and $N_{S}$. Evidently they depend linearly upon the flux normalizations and have a mild dependence upon the assumed slope $\alpha$. The observed number of events can be treated in Poisson statistics and thus compared with the expectations.

The first comparison between data and observations uses the track-to-shower ratio, $N_{T} / N_{S}$ [6]. This is a very neat way to display IceCube's observations, however, the predictions depend a bit upon the slope [4]. The second manner uses the fact that in an equilateral triangle, the sum of the 3 distances of a inner point from the sides is constant (=Viviani theorem) applied to the 3 fraction of fluxes, e.g. $F_{v_{e}} /\left(F_{v_{e}}+F_{v_{u}}+F_{v_{\tau}}\right)$ for the electronic fraction [7]. This is called flavor triangle and it is used e.g. in [8], see [5] for the study of the theoretical errors. In this way, the predictions become independent from the slope of the power law but the observations are not; moreover, the fractions of the fluxes are not directly observable. Expectations and observations are found to be in agreement, as evident from Fig. 1. This is true in particular for the conventional mechanism of neutrinos production, i.e. pion decay. The speculative cases when neutrinos at the source derive from neutron decay are somewhat disfavored, while the flavor ratio expected from prompt events in atmosphere is not, instead.

These results are significant extensions of the directly available knowledge. In fact, vacuum oscillations become perceivable when the product of the difference of neutrino masses squared $\Delta m^{2}$ times the distance between production and detection $L$ times the inverse of the neutrino energy $E_{v}$ obeys the condition: $\Delta m^{2} c^{4} L /\left(h c E_{v}\right) \gg 1$ i.e., $0.81 \Delta m^{2} c^{4} / \mathrm{eV}^{2} L / \mathrm{km} /\left(E_{v} / \mathrm{GeV}\right) \gg 1$, obtained from the $1^{\text {st }}$ oscillation maximum. This is less than few hundreds for atmospheric neutrinos crossing the whole Earth and with $E_{v}=100 \mathrm{MeV}$. For a hypothetical source of the Milky Way, this is at least $10^{6}$, even if we use the smallest $\Delta m^{2}$ with $L=1 \mathrm{kpc}$ and $E_{v}=1 \mathrm{PeV}$. Accepting the most plausible option, 
Table 1. Expected number of events per year in IceCube as a function of the spectral index [10].

\begin{tabular}{|c|c|c|c|}
\hline & $\alpha=2$ & $\alpha=2.3$ & $\alpha=2.6$ \\
\hline Double pulse & 0.2 & 0.1 & $<0.1$ \\
\hline Glashow $p p$ & 0.5 & 0.2 & $<0.1$ \\
\hline Glashow $p \gamma$ & 0.2 & 0.1 & $<0.1$ \\
\hline
\end{tabular}

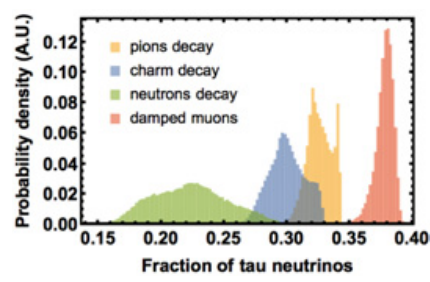

Figure 2. The amount of tau neutrinos depends a little bit on the production mechanism and it is not perfectly known due to residual uncertainties in oscillations parameters, but the main point to emphasize is simply that: If tau neutrinos are observed, the cosmic origin is proved.

that these neutrinos are extragalactic, the distances increase at least by $10^{3}$ times and consequently also the product! This means that we have attempted to rely on what we have learned in the terrestrial laboratories by endorsing a brave extrapolation... and, till now, it works.

\section{Inferences on cosmic neutrinos}

We elaborate on hypothesis cosmic neutrino. A large amount of tau neutrinos should be present, and if the spectrum does not deviate strongly from a power law, the Glashow resonance signal will be observable, and its amount will inform us on the type of cosmic source; see Table 1 for a summary. Moreover, we can begin to explore (or exclude) exotic possibilities such as neutrino decay.

Tau neutrinos:- To date, no tau event has been observed in the existing dataset but the expectations have been clarified $[9,10]$. Due to oscillations tau neutrinos are unavoidable [3], see Fig. 2. A tau neutrino below few $100 \mathrm{TeV}$ produces a signal similar to the one produced by an electron neutrino or a neutral current event. At high energies, the primary vertex (where hadrons deposit energy) and the secondary vertex (where tau decays) are separated; this is the same principle used by the OPERA detector on a microscopic scale. If the energy is larger than few $\mathrm{PeV}$, the two vertices are visible in two separated sets of phototubes, and this is called double-bang [3]. The single phototubes of IceCube, able to resolve the time structure of the energy deposited, can identify a double-pulse [9] already above half a PeV. Double pulse events are expected to be contributed from a region where we have already observed neutrinos: If neutrinos are power law distributed, we are confident at 90\% CL to observe a tau when the exposure of IceCube will be tripled, i.e., in about 10 years.

Glashow resonance:- The production of real $W^{-}$bosons by $\bar{v}_{e}$ impinging on electrons, i.e., the Glashow resonance, requires antineutrinos of $6.32 \mathrm{PeV}$, namely with energies 3 times larger than the highest one to date observed. Interestingly, given the observed signal at lower energies, the intensity of Glashow resonance does depend upon the production mechanism; indeed, if we have $p \gamma$ collisions, we produce mostly $\pi^{+}$and thus $\bar{v}_{e}$ arise only when we consider neutrino oscillations [11, 12]. The signal is about two times less in this case than for $p p$ collisions, see e.g. [5]. Again, no signal is observed yet. Plausible explanations are, $i$ ) the spectral index is larger than $2 ; i i$ ) the slope of the cosmic neutrino component is close to 2, but we have another component with larger slope; $i \mathrm{i} i$ ) there is a cutoff in the spectrum before $6 \mathrm{PeV} ; i v$ ) or we need to collect more exposure. The calculations of [10] authorize a moderate optimism 
toward the possibilities to observe a signal, even if it emphasizes the difficulties to distinguish the $p \gamma$ and $p p$ cases; compare with the conclusions of $[12,13]$.

Neutrino decay:- The idea that neutrinos could be unstable is old. A radical possibility is that the heavier neutrinos decay to the lighter one and an almost invisible, massless particle. After IceCube data, this hypothesis has been investigated, relying again on the obseved track-to-shower ratio. Since the lightest neutrino mass has a different composition in the normal hierarchy and the inverse hierarchy, the predictions are different. More precisely, in the first case the probability to receive a muon neutrinos $\left|U_{\mu 1}^{2}\right|$ is smaller than in the usual case, whereas in the second case, this probability is $\left|U_{\mu 3}^{2}\right|$ that is, instead, larger than in the usual case. By using the four years data [14], it was shown that in any case in both mass hierarchies, we can claim that this hypothesis on neutrino decay is disfavored at least at the $2 \sigma \mathrm{CL}$. Note incidentally that an important additional information derives from the neutrinos from SN1987A, whose mere detection witnesses against inverse mass hierarchy, since the lightest antineutrino $\bar{v}_{3}$ contains a very tiny amount of the species that was detected, namely, $\bar{v}_{e}[15]$.

\section{Prospects for astronomical identification}

Neutrino-gamma connection at the source:- The most reasonable hypothesis for the origin of cosmic neutrinos is that they come from regions of the Universe where cosmic rays energies collide with gas or with some photonic target, producing copiously mesons. In first approximation, the collisions with gas will produce $\pi^{+}, \pi^{-}, \pi^{0}$ in similar proportions whereas the collisions with photons $p \gamma \rightarrow \Delta^{+}$will produce two $\pi^{0}$ per $\pi^{+}$, owing to isospin selection rules. The charged pions, upon decay, yield in turn neutrinos but we expect to have also gamma rays from $\pi^{0}$ decay. We conclude that,

$\gamma$-rays are four times more abundant for $p \gamma$ sources than for $p p$ sources

Moreover, a quantitative estimation of the connection of $\gamma$-ray and neutrino fluxes [16], assuming $p p$ sources, shows that the two are quite similar (equal within $20 \%$ or so depending on the slope).

Destiny of $\gamma$-rays and the window of Fermi-LAT:- Neutrinos and $\gamma$-rays differ, since the latter might interact after being produced. E.g., collisions with the photons that permeate the Universe lead to $e^{+} e^{-}$ production, for energies above $E_{\gamma} \approx m_{e}^{2} / \epsilon_{\gamma}=250 \mathrm{GeV}$ if $\epsilon_{\gamma}=1 \mathrm{eV}$. Thus, if the source is transparent and the leptonic emission is not overwhelming, there is a chance to observe $\gamma$-rays directly produced with neutrinos. It is in the energy window below $100 \mathrm{GeV}$ or so, studied by Fermi-LAT. The very high energy $\gamma$-rays that correspond to IceCube's neutrinos are necessarily modified, if not else, by their propagation over cosmological distances. They will be degraded till they reach energies below $100 \mathrm{GeV}$ or so, when Universe is (approximatively) transparent. These $\gamma$-rays, once again, contribute to the window of energy monitored by Fermi-LAT.

BL Lacertae objects as neutrino sources:- We have argued the importance of $\gamma$-ray observations below $100 \mathrm{GeV}$. Fermi-LAT proved that the BL Lac are the brightest extragalactic sources above $10 \mathrm{GeV}$. These are AGNs with jets pointing toward the Earth and featureless optical spectra (i.e., lacking strong emission/absorption lines). Many more of these BL Lac are not visible as point sources, being too faint and/or too far from us. They contribute to the unresolved $\gamma$-ray radiation, again observed by Fermi-LAT see Table 2. The sum of resolved and unresolved BL Lac almost saturates the observed diffuse emission, with a margin of uncertainty of some ten percents, that should be attributed to other sources. Figure 3 compares the total BL Lac $\gamma$-rays spectrum with the one of neutrinos.

The angular distribution:- In first approximation, IceCube's events are distributed isotropically but a new significant component with a different distribution, possibly correlated with some galactic structure, 
Table 2. The $1^{\text {st }}$ column is obtained summing the intensity of 211 identified BL Lac [17] (5 BL Lac have more than $1 \%$ of the total $\gamma$-ray flux each; the brightest has $2 \%$ ); the $2^{\text {nd }}$ extrapolates to the low galactic latitude region $|b|<15^{\circ}$, avoided in the analysis, by assuming isotropy; the last two are theoretical estimations available from [17]. We checked that models in [17] imply total numbers in reasonable agreement with those obtained summing resolved and unresolved BL Lac. The theoretical models named LDDE and PLE are defined in [17].

\begin{tabular}{|c||c|c|c|c|}
\hline BL Lac class & resolved & resolved (all) & unresolved (LDDE) & unresolved (PLE) \\
\hline Flux $10^{-7} \mathbf{p h} /\left(\mathbf{c m}^{2} \mathbf{s ~ s r}\right)$ & 6.3 & 8.5 & $8_{-1.3}^{+2.0}$ & $10_{-1.7}^{+2.1}$ \\
\hline
\end{tabular}

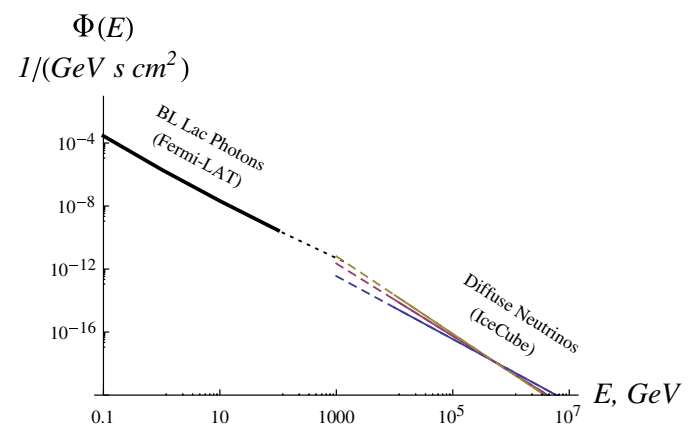

Figure 3. Total emission in $\gamma$-rays from BL Lac, as summarized by the best fit LDDE model [17], compared with the neutrino emission of IceCube for the 3 representative fits of [18]. Dotted and dashed lines are simply extrapolations. There is no strong incompatibility with the hypothesis that $\gamma$ and neutrino emissions come from BL Lac, but the shape of the spectra is quite different especially if the neutrino spectrum is steep. The importance to understand the neutrino emission at the lowest energies is evident.

is not excluded. No evident point source or class of sources corresponds to these events. The direction of the showers is identified with a precision of $\delta \theta=10-20^{\circ}$, whereas the tracks are known with an angular resolution 10 times better. This is important, since the fraction of solid angle is proportional to the square of $\delta \theta,{ }^{1}$ despite the fact that the tracks are less and subject to background contamination especially at low energies.

\section{Unconventional sources}

Motivated by the IceCube events, several new physics scenarios have been proposed as possible sources of these events, whether the entirety or at least a part of the events. Among them the scenario of interpreting the events by decaying $\mathrm{PeV}$-scale dark matter gained attention. In this scenario the neutrinos are originated from the Galactic and extra-galactic components of the flux from the dark matter decay to standard model particles, say leptons and quarks, with the lifetime $\sim 10^{28} \mathrm{~s}$ [19]. Figure 4 shows the energy distribution of events in dark matter scenario. The angular distribution of the events also marginally prefer the expected distribution in dark matter scenarios rather than the pure isotropic extragalactic astrophysical scenario [20]. However, larger statistics are required to make any conclusion. The dark matter scenario can be tested in the near future by high energy gamma-ray experiments, both by the IGRB data [21], or extensive air-shower detectors [22]. Particularly, in this scenario an accompanying flux of high energy gamma-rays with peculiar anisotropy pattern is expected, at a level detectable by near future detectors, such as LHAASO [23].

\footnotetext{
${ }^{1}$ For this reason, high energy neutrino telescopes in water have a strong scientific advantage, being able to obtain $\delta \theta \approx 0.2^{\circ}$.
} 


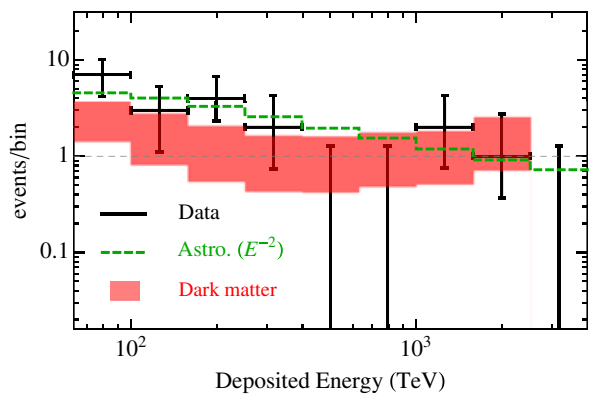

Figure 4. The shaded region shows the energy distribution of events in a toy model for decaying dark matter scenario. The width of the shaded regions corresponds to variation of dark matter lifetime within $1 \sigma$ range around the best-fit point obtained from the fit to IceCube data. The dark matter mass assumed equal to $4 \mathrm{PeV}$. The green dashed line shows the expected events from astrophysical $E_{v}^{-2}$ flux. The black points show the IceCube data.

\section{Discussion}

The case made by IceCube for a new population of very high energy neutrinos, on top of conventional atmospheric neutrinos is very convincing, even if the role of charm (prompt) contribution is still to be elucidated. The new population can be attributed to cosmic neutrinos, that are searched since more than half a century. It is consistent with ordinary vacuum oscillations. A definitive proofs of this hypothesis could come from tau and/or Glashow resonance. The conventional expectation of a hard $\sim E^{-2}$ distributions would imply that they are observable; tau events are less but largely contributed by neutrinos of the energies that have been already seen. However, a fit of the IceCube data with a power law distribution prefers steeper slopes. The main needs are: to confirm the case for cosmic neutrinos independently; to search deviations from an isotropic distribution; to locate (some of) its source(s); to understand well the spectrum; to remind that this could be just the beginning.

\section{References}

[1] S. Schonert, T.K. Gaisser, E. Resconi and O. Schulz, Phys. Rev. D 79 (2009) 043009

[2] R. Enberg, M.H. Reno and I. Sarcevic, Phys. Rev. D 78 (2008) 043005

[3] J.G. Learned and S. Pakvasa, Astropart. Phys. 3 (1995) 267

[4] A. Palladino, G. Pagliaroli, F. L. Villante and F. Vissani, Phys. Rev. Lett. 114 (2015) 17, 171101

[5] A. Palladino and F. Vissani, Eur. Phys. J. C 75 (2015) 433

[6] A. Esmaili and Y. Farzan, Nucl. Phys. B 821 (2009) 197

[7] G. Barenboim and C. Quigg, Phys. Rev. D 67 (2003) 073024

[8] M.G. Aartsen et al. [IceCube Collaboration], Phys. Rev. Lett. 114 (2015) 17, 171102

[9] M.G. Aartsen et al. [IceCube Collaboration], arXiv: 1509.06212

[10] A. Palladino, G. Pagliaroli, F.L. Villante and F. Vissani, arXiv: 1510.05921

[11] V.S. Berezinsky and A.Z. Gazizov, JETP Lett. 25 (1977) 254

[12] L. A. Anchordoqui, H. Goldberg, F. Halzen and T.J. Weiler, Phys. Lett. B 621 (2005) 18

[13] V. Barger, J. Learned and S. Pakvasa, Phys. Rev. D 87 (2013) 3, 037302

[14] G. Pagliaroli, A. Palladino, F. Vissani and F.L. Villante, arXiv:1506.02624, accepted in PRD

[15] P. Baerwald, M. Bustamante and W. Winter, JCAP 1210 (2012) 020

[16] F.L. Villante and F. Vissani, Phys. Rev. D 78 (2008) 103007

[17] M. Ajello et al. [Fermi-LAT Collaboration], Astrophys. J. 780 (2014) 73

[18] T.K. Gaisser [IceCube Collaboration], arXiv: 1507.07871 
[19] A. Esmaili and P.D. Serpico, JCAP 1311 (2013) 054

[20] A. Esmaili, S.K. Kang and P.D. Serpico, JCAP 1412 (2014) 054

[21] K. Murase, R. Laha, S. Ando and M. Ahlers, Phys. Rev. Lett. 115 (2015) 071301

[22] A. Esmaili and P.D. Serpico, JCAP 1510 (2015) 014

[23] S. Vernetto and P. Lipari, http://pos.sissa.it/archive/conferences/236/740/ICRC 2015_740.pdf 